\title{
Therapeutic Drug Monitoring of Quetiapine: Effect of Coadministration with Antiepileptic Drugs in Patients with Psychiatric Disorders
}

\author{
Vincenza Santoro ${ }^{1,2}$, Concetta D'Arrigo ${ }^{1,2}$, Gaetana Migliardi ${ }^{1}$, Maria Rosaria Muscatello ${ }^{3}$, Umberto \\ Micò $^{3}$, Rosario Cambria ${ }^{3}$ and Edoardo Spina ${ }^{1,2, *}$
}

${ }^{I}$ Section of Pharmacology, Department of Clinical and Experimental Medicine and Pharmacology, ${ }^{2}$ IRCCS Centro Neurolesi "Bonino-Pulejo" and ${ }^{3}$ Department of Neurosciences, Psychiatric and Anesthesiological Sciences, University of Messina, Messina, Italy

\begin{abstract}
Antiepileptic agents, particularly those with mood-stabilizing properties, are increasingly used in the management of psychiatric disorders and may be prescribed in combination with antipsychotics. Aim of the present study was to evaluate the pharmacokinetic interaction between various antiepileptics and quetiapine, a second-generation antipsychotic, in psychiatric patients, by using data from a therapeutic drug monitoring service. Steady-state plasma concentrations of quetiapine were compared in patients treated with quetiapine alone (controls, $n=35$ ) and in patients comedicated with valproic acid $(n=19)$, lamotrigine $(n=16)$, carbamazepine $(n=6)$, topiramate $(n=6)$ and oxcarbazepine $(n=5)$. The six groups were matched for sex, age and daily dose of quetiapine. Dose-normalized plasma concentrations of quetiapine were significantly lower in the carbamazepine group than in the control group $(\mathrm{p}<0.001)$, while there were no differences in plasma quetiapine concentrations between the valproic acid, lamotrigine, topiramate or oxcarbazepine groups and the control group. In 5 patients assessed on and off carbamazepine comedication, dose-normalized plasma concentrations of quetiapine were significantly lower during combination therapy than on quetiapine alone $(\mathrm{p}<0.01)$. By contrast, no appreciable changes in plasma levels of quetiapine were found in the 8 and 6 patients assessed on and off valproic acid or lamotrigine comedication, respectively. These findings confirm that carbamazepine decreases markedly steady-state plasma concentrations of quetiapine, presumably by inducing its CYP3A4-mediated biotransformation. Conversely, concomitant administration of therapeutic doses of valproic acid, lamotrigine, topiramate or oxcarbazepine does not appear to affect significantly plasma levels of quetiapine.
\end{abstract}

Key Words: Quetiapine, carbamazepine, valproic acid, lamotrigine, topiramate, oxcarbazepine, drug interaction, CYP3A4.

\section{INTRODUCTION}

Quetiapine, a dibenzothiazepine derivative, is a secondgeneration antipsychotic effective in the treatment of schizophrenia and bipolar disorder [1-3]. Quetiapine is an antagonist at a broad range of neurotransmitter receptors [1, 4]. It has a modest affinity for dopamine D2 receptors, to which it shows only a transiently high occupancy, and a higher affinity for serotonin $5-\mathrm{HT}_{2 \mathrm{~A}}, \alpha_{1}$-adrenergic and histaminergic receptors, while it has limited affinity for muscarinic receptors. Furthermore, quetiapine is a partial agonist at $5-\mathrm{HT}_{1 \mathrm{~A}}$ receptors [5].

Multiple dose pharmacokinetic studies have demonstrated that quetiapine exhibits linear pharmacokinetics in a dose range up to $750 \mathrm{mg} /$ day [6]. Quetiapine is extensively metabolized in the liver and eleven confirmed metabolites have been identified [6]. Two of these, 7-hydroxy-quetiapine and 7-hydroxy-N-desalkyl-quetiapine, possess pharmacological activity. However, as they are found only at low concentrations in plasma, it is unlikely that they contribute to

\footnotetext{
*Address correspondence to this author at the Department of Clinical and Experimental Medicine and Pharmacology, Section of Pharmacology, University of Messina, Policlinico Universitario di Messina, Via Consolare Valeria, 98125 Messina, Italy; Tel: +39 090 2213647; Fax: +39 090 2213300; E-mail: espina@unime.it
}

clinical effects of quetiapine. The biotransformation of quetiapine is mediated mainly by the cytochrome P450 (CYP) 3A4 and, to a lesser extent, by CYP2D6 [6]. Therefore, coadministration of inhibitors or inducers of CYP3A4 may interfere with quetiapine elimination, thereby resulting in potentially significant pharmacokinetic interactions. In this respect, coadministration of drugs that are known to induce CYP3A4, such as phenytoin and carbamazepine may lead to a clinically relevant decrease in quetiapine concentrations $[7,8]$. On the other hand, ketoconazole, an inhibitor of CYP3A4, increases quetiapine concentrations and decrease its clearance [8].

Like other second-generation antipsychotics, quetiapine may be prescribed in combination with antiepileptic agents, particularly those with mood stabilizing properties including carbamazepine, valproic acid and lamotrigine [9]. Therefore, information on potential pharmacokinetic interactions between these compounds is important for safe prescribing. As previously mentioned, it is well documented that carbamazepine, an inducer of CYP3A4, may decrease plasma concentrations of quetiapine [8]. There are controversial findings concerning the effect of valproic acid on quetiapine elimination. Plasma concentrations of quetiapine were found to be either unchanged or increased in psychiatric patients during coadministration with valproic acid [10-12]. There is 
limited or no information concerning the effect of lamotrigine and other antiepileptic agents that are also occasionally used to treat psychiatric disorders, such as topiramate and oxcarbazepine, on the pharmacokinetics of quetiapine [11, 13].

Therapeutic drug monitoring (TDM) has been regarded as a valid tool to optimise pharmacotherapy in psychiatry [14]. In order to investigate the potential interaction between quetiapine and various antiepileptics, we analyzed plasma concentrations of quetiapine in psychiatric patients, derived from our routine TDM service.

\section{PATIENTS AND METHODS}

\section{Patients}

Since January 2006, determination of plasma quetiapine concentration was included in our routine TDM service. Samples from patients treated with quetiapine alone or in combination with antiepileptic drugs over a period of 2 years (January 2006-December 2007) were considered for the study. In our TDM service blood samples are taken after at least one week on the same dose to assure steady-state concentrations. Samples for which the request forms did not include the daily dose of the antipsychotic and concomitant antiepileptic medications, time for the last dose and time of blood sampling were excluded. Samples were excluded if the patient was given concomitantly drugs known to inhibit or induce CYP3A4, with the exception of carbamazepine. Following these procedures, the final data set consisted of 138 plasma concentrations values from 87 psychiatric patients (49 females and 38 males, aged 23 to 60 years), most suffering from bipolar or schizoaffective disorder. The 87 patients were divided into 6 groups with respect to antiepileptic comedication: quetiapine monotherapy $(n=35)$, quetiapine in combination with valproic acid $(n=19)$, quetiapine with lamotrigine $(n=16)$, quetiapine with carbamazepine $(n=6)$, quetiapine with topiramate $(n=6)$, and quetiapine with oxcarbazepine $(n=5)$. When multiple samples were available from the same patient, only the first plasma concentration value was considered for the cross-sectional data analysis.

In 8 patients from the valproic acid group, in 6 from the lamotrigine and in 5 patients from the carbamazepine group, plasma quetiapine concentrations could be also assessed before addition of antiepileptic comedication or at least 4 weeks after its discontinuation.

Blood samples were drawn into heparinized tubes at $08.00 \mathrm{~h}$ (approximately 12 to 13 hours after the last dose of quetiapine). The plasma was kept frozen at $-20^{\circ} \mathrm{C}$ until assayed for quetiapine.

The study protocol was approved by the Ethics Committee of Messina University Hospital.

\section{Analytical Methods}

Quetiapine was analyzed by a HPLC method developed in our laboratory. Briefly, to $1.0 \mathrm{ml}$ plasma in a $15-\mathrm{ml}$ tube were added $15 \mu \mathrm{l}$ of the working internal standard (IS) solution (clozapine $10 \mu \mathrm{g} / \mathrm{ml}$ ) and $1 \mathrm{ml}$ of $\mathrm{NaOH} 1 \mathrm{~mol} / \mathrm{l}$. The tubes were briefly mixed with a vortex and $4 \mathrm{ml}$ of extraction solvent (90:10 vol/vol $n$-hesane- diisopropyl ether) were added to samples. After 15 minutes of shaking at $300 \mathrm{cy}-$ $\mathrm{cles} / \mathrm{min}$, the mixture was centrifuged at $3000 \mathrm{~g}$ for $10 \mathrm{~min}$ - utes. The organic phase was transferred to tubes containing $150 \mu \mathrm{l}$ of phosphate buffer $0.1 \mathrm{~mol} / 1 \mathrm{pH} 2.2$, mixed at 300 cycles/min for 15 minutes and centrifuged at $3000 \mathrm{~g}$ for 10 minutes. The organic layer was carefully discarded and $50 \mu 1$ of the remaining aliquot $(150 \mu \mathrm{l})$ was injected into the HPLC system. The chromatographic equipment was purchased from Shimadzu (Kyoto, Japan) and consisted of a Model LC $10 \mathrm{Advp}$ dual reciprocating pump coupled to a manual injector (Rheodyne 7725i) with a $50 \mu$ fixed loop, a Model SPD $10 \mathrm{AV}$ UV-Vis detector with variable wavelength set at 278 $\mathrm{nm}$ and a Model SCL 10A System Controller. The chromatographic separation was performed using a $\mathrm{C}_{18}$ BDSHypersil analytical column $(3 \mu \mathrm{m}, 100 \mathrm{~mm} \times 4.6 \mathrm{~mm}$ ID). The mobile phase consisted of water-acetonitrile-methanol 70:25:5 vol $/ \mathrm{vol} / \mathrm{vol}$, containing triethylamine $0.3 \%$ and 0.05 mol/1 $\mathrm{KH}_{2} \mathrm{PO}_{4} \mathrm{pH} 2.53$, adjusted with $\mathrm{H}_{3} \mathrm{PO}_{4}(25 \%)$. The flow rate was $1 \mathrm{ml} / \mathrm{min}$ at ambient temperature with an operating pressure of $12.0 \mathrm{MPa}$. The run time of the assay was 8 minutes and the drugs are well resolved with retention times of 4.6 for quetiapine and 6.7 minutes for clozapine. The method had a calibrated range of 5-600 ng/ml and linearity was shown through this interval. Recovery was determined at 7 different concentrations from 5 to $600 \mathrm{ng} / \mathrm{ml}$ and ranged from 80.8 to $96.5 \%(89.6 \pm 5.7 \%$; mean $\pm S D ; n=5)$. Recovery of clozapine was $89.6 \pm 4.1 \%$. Precision of the method was quantified by calculating intraday and interday coefficients of variation (CVs). Within the range of concentrations investigated, intraday and interday CVs were less than $11.0 \%$ and $8.0 \%$, respectively. Accuracy was expressed as percent error $(\%)$ which ranged from $-0.6 \%$ to $17.6 \%$. The limit of detection and the limit of quantification for quetiapine were $2 \mathrm{ng} / \mathrm{ml}$ and $5 \mathrm{ng} / \mathrm{ml}$, respectively.

As a measure of compliance with the most frequently coadministered antiepileptic medications, plasma concentrations of valproic acid and carbamazepine were determined by enzyme-multiplied immunoassay $\left(\mathrm{EMIT}^{\mathrm{R}}\right)$, while plasma levels of lamotrigine were determined by the HPLC method for risperidone described by Avenoso et al. [15] that allows co-extraction of lamotrigine. The limit of quantification for lamotrigine was $0.05 \mu \mathrm{g} / \mathrm{ml}$.

\section{Statistical Analysis}

Analysis of variance was used to compare demographic and dosage data among the groups. Because of the wide range of quetiapine doses used in clinical settings, plasma concentrations of quetiapine were normalized for the dose by dividing the measured concentration $(\mathrm{ng} / \mathrm{ml})$ by the daily administered dose (mg). As plasma concentrations of quetiapine were not normally distributed, medians rather than means were calculated for the corresponding data sets and comparisons were carried out by using the Kruskal-Wallis non-parametric rank analysis of variance. The MannWhitney U-test was used for two-group comparisons, while the Wilcoxon signed-rank test was used for intraindividual comparisons. A p value of 0.05 or less was regarded as significant.

\section{RESULTS}

In the 87 patients included in the study, quetiapine dose ranged from 50 to $900 \mathrm{mg} /$ day (mean dose $\pm \mathrm{SD}$ : $366 \pm 221$ $\mathrm{mg} /$ day; median: $350 \mathrm{mg} /$ day). Plasma concentrations of 
quetiapine ranged from 5 to $484 \mathrm{ng} / \mathrm{ml}$. Basic demographic data and dose-normalized plasma concentrations of quetiapine in the six groups are summarized in Table 1. The six groups were matched for age, sex and daily dose of quetiapine (Table 1).

Dose-normalized steady-state plasma concentrations of quetiapine differ significantly among groups $(\mathrm{p}<0.05)$. When comparing each of the 5 groups on antiepileptic comedication with the quetiapine monotherapy group (control group), dose-normalized plasma concentrations of quetiapine were significantly lower in the carbamazepine group than in the control group $(p<0.001)$, while there were no differences in plasma quetiapine concentrations between the valproic acid, lamotrigine, topiramate or oxcarbazepine groups and the control group.

Since quetiapine dosage was modified in the subgroups assessed on and off comedication, pharmacokinetic comparisons in these patients were also based on dose-normalized data. In the 5 patients assessed on and off carbamazepine comedication, plasma levels (means \pm SD) of quetiapine were significantly lower during combination therapy than on quetiapine alone $(0.05 \pm 0.02$ vs $0.22 \pm 0.07 \mathrm{ng} / \mathrm{L}$ per $\mathrm{mg}$, $\mathrm{p}<0.01)$. By contrast, no appreciable changes in plasma levels of quetiapine were found in the 8 and 6 patients assessed on and off valproic acid or lamotrigine comedication, respectively. Dose-normalized plasma concentrations of quetiapine in individual patients assessed on and off comedication with carbamazepine, valproic acid or lamotrigine are shown in Fig. (1).

Plasma concentrations of valproic acid ranged from 30 to $91 \mu \mathrm{g} / \mathrm{ml}$, plasma concentrations carbamazepine from 5.2 and $10.7 \mu \mathrm{g} / \mathrm{ml}$, while those of lamotrigine ranged from to 0.8 to $5.1 \mu \mathrm{g} / \mathrm{ml}$.

\section{DISCUSSION}

In view of the increasing use of antiepileptic agents in combination with new antipsychotics for the treatment of bipolar or schizoaffective disorder, the possibility of a phar- macokinetic interaction between these compounds needs to be investigated $[16,17]$.

In the present study, by using data from a routine TDM service, plasma concentrations of quetiapine were found to be significantly lower (by approximately 80\%) in patients concomitantly treated with carbamazepine as compared to patients on quetiapine monotherapy or receiving comedication with other antiepileptic agents such as valproic acid, lamotrigine, topiramate and oxcarbazepine. These findings from the cross-sectional investigation were reinforced by the results from subgroups of patients evaluated in the absence and in presence of antiepileptic comedication. In the five patients assessed on and off carbamazepine comedication, plasma concentrations of quetiapine decreased when carbamazepine was added or increased when it was discontinued, while no major modifications in quetiapine levels were observed in patients with or without valproic acid ( 8 pts.) or lamotrigine (6 pts.).

In agreement with previous evidence from pharmacokinetic and drug monitoring studies [8, 11, 18], our findings confirm that carbamazepine may cause a prominent decrease in plasma concentrations of quetiapine. The change in antipsychotic drug levels during carbamazepine coadministration is likely explained by enzyme induction. Carbamazepine is a potent inducer of various CYPs including CYP3A4 which is the major isoform involved in the biotransformation of quetiapine [19]. In this respect, carbamazepine has been reported to reduce the plasma concentrations of a number of first-generation (i.e., haloperidol and chlorpromazine) and second-generation antipsychotics (i.e., clozapine, risperidone, olanzapine and ziprasidone) [20, 21]. Admittedly, as quetiapine is also metabolized via CYP2D6, the inducing effect of carbamazepine might be mediated, at least partially, by CYP2D6. However, in contrast to all other CYPs involved in drug metabolism, CYP2D6 does not appear to be inducible [22]. The marked decrease in antipsychotic levels caused by carbamazepine may have important clinical implications as it could result in decreased therapeutic efficacy. Larger doses of quetiapine may be required to achieve or to

Table 1. Demographic Data and Plasma Concentrations of Quetiapine in Patients Treated with Quetiapine Alone (Controls, $n=35)$ or in Combination Valproic Acid $(n=19)$, Lamotrigine $(n=16)$, Carbamazepine $(n=6)$, Topiramate $(n=6)$ or Oxcarbazepine $(n=5)$. Results are Expressed as Means \pm SD or Medians (Ranges are in Brackets)

\begin{tabular}{|c|c|c|c|c|c|c|}
\hline & $\begin{array}{c}\text { Quetiapine Mono- } \\
\text { therapy } \\
(\mathbf{n}=35)\end{array}$ & $\begin{array}{l}\text { Quetiapine and } \\
\text { Valproic Acid } \\
\qquad(\mathbf{n}=19)\end{array}$ & $\begin{array}{l}\text { Quetiapine and } \\
\text { Lamotrigine } \\
\quad(n=16)\end{array}$ & $\begin{array}{l}\text { Quetiapine and } \\
\text { Carbamazepine } \\
\qquad(n=6)\end{array}$ & $\begin{array}{l}\text { Quetiapine and } \\
\text { Topiramate } \\
(n=6)\end{array}$ & $\begin{array}{l}\text { Quetiapine and } \\
\text { Oxcarbazepine } \\
\qquad(n=5)\end{array}$ \\
\hline Men/women & $16 / 19$ & $8 / 11$ & $7 / 9$ & $3 / 3$ & $2 / 4$ & $2 / 3$ \\
\hline Age (yrs) & $39.3 \pm 8.8$ & $39.4 \pm 9.4$ & $41.3 \pm 9.1$ & $40.8 \pm 11.3$ & $41.5 \pm 9.8$ & $42.8 \pm 10.4$ \\
\hline $\begin{array}{c}\text { Daily dose of } \\
\text { quetiapine }(\mathrm{mg})\end{array}$ & $\begin{array}{c}378 \pm 226 \\
(50-900)\end{array}$ & $\begin{array}{c}343 \pm 232 \\
(50-800)\end{array}$ & $\begin{array}{c}355 \pm 205 \\
(75-800)\end{array}$ & $\begin{array}{l}458 \pm 280 \\
(100-900)\end{array}$ & $\begin{array}{c}342 \pm 235 \\
(50-750)\end{array}$ & $\begin{array}{c}315 \pm 169 \\
(75-500)\end{array}$ \\
\hline $\begin{array}{c}\text { Daily dose of } \\
\text { antiepileptic (mg) }\end{array}$ & - & $\begin{array}{c}958 \pm 271 \\
(500-1500)\end{array}$ & $\begin{array}{c}194 \pm 54 \\
(100-300)\end{array}$ & $\begin{array}{c}733 \pm 207 \\
(400-1000)\end{array}$ & $\begin{array}{c}208 \pm 66 \\
(100-300)\end{array}$ & $\begin{array}{l}1080 \pm 164 \\
(900-1200)\end{array}$ \\
\hline $\begin{array}{c}\text { Dose-normalized plasma } \\
\text { concentrations of quetiapine } \\
\qquad(\mathrm{ng} / \mathrm{ml}) /(\mathrm{mg})^{\mathrm{a}}\end{array}$ & $\begin{array}{c}0.16 \\
(0.06-0.74)\end{array}$ & $\begin{array}{c}0.17 \\
(0.08-0.65)\end{array}$ & $\begin{array}{c}0.17 \\
(0.07-0.43)\end{array}$ & $\begin{array}{c}0.04^{\mathrm{b}} \\
(0.01-0.07)\end{array}$ & $\begin{array}{c}0.16 \\
(0.12-0.24)\end{array}$ & $\begin{array}{c}0.15 \\
(0.11-0.27)\end{array}$ \\
\hline
\end{tabular}

${ }^{a}$ Significant difference among groups $(\mathrm{p}<0.05$, Kruskal-Wallis test)

${ }^{\mathrm{b}} \mathrm{p}<0.001$ vs quetiapine monotherapy (Mann-Whitney U-test). 

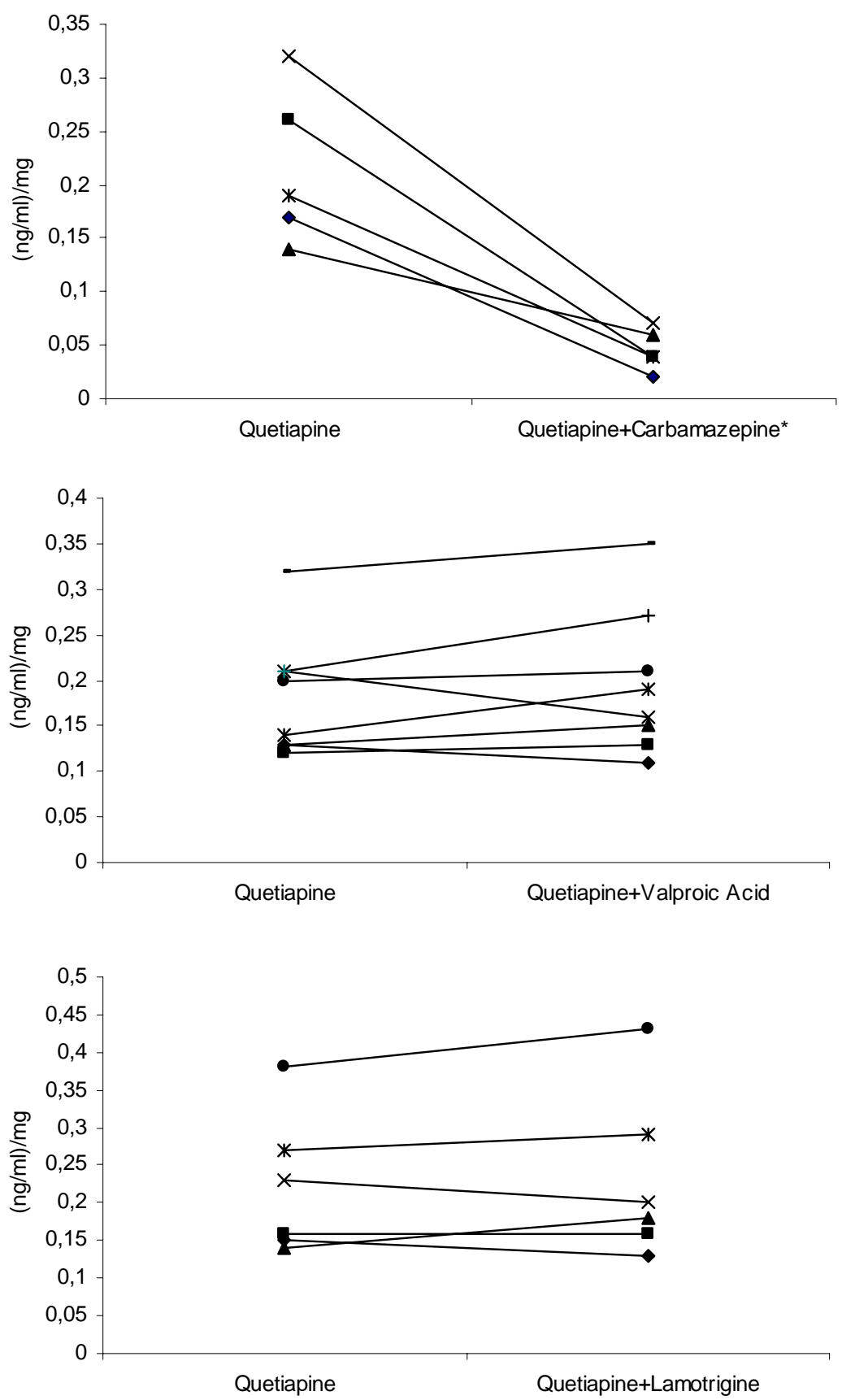

Fig. (1). Dose-normalized plasma concentrations of quetiapine in individual patients assessed on and off comedication with carbamazepine (upper panel), valproic acid (middle panel) or lamotrigine (lower panel). ${ }^{*} \mathrm{p}<0.01$ vs off-comedication period (Wilcoxon signed-rank test).

maintain antipsychotic effect when the drug is used in combination with carbamazepine. Interestingly, in this study, patients of the carbamazepine group were prescribed a quetiapine dose slightly higher than other groups.

In the current study, concomitant treatment with valproic acid, given at dosages up to $1500 \mathrm{mg} /$ day, had minimal and not significant effects on plasma concentrations of quetiapine. Aichorn et al. [12] reported that comedication with valproate in 9 patients was associated with a $77 \%$ increase in plasma levels of quetiapine, an effect that was attributed to inhibition of CYP3A4. Although valproic acid is a broadspectrum metabolic inhibitor, in vitro studies in human liver microsomes have documented that it is only a weak inhibitor of CYP3A4 [23]. Consistent with our findings and this biochemical evidence, data from a formal kinetic investigation in psychiatric patients [10] and from a routine TDM service [11] have indicated that valproic acid does not affect significantly quetiapine pharmacokinetics. 
Differently from the results of the present investigation, a TDM study reported that plasma quetiapine concentrations were slightly, but significantly reduced (by 17\%) in patients comedicated with lamotrigine [11]. Unlike many other AEDs, lamotrigine does not appear to inhibit or induce CYP enzymes [24]

Though the limited number of samples in the topiramate and oxcarbazepine groups does not allow definite conclusions, our findings indicate that topiramate and oxcarbazepine are unlikely to cause clinically relevant modifications of plasma concentrations of quetiapine. Topiramate is a weak inducer of CYP3A4 as it may decrease plasma concentrations of steroid oral contraceptives [25]. In agreement with the actual findings, a previous study from our group reported that adjunctive topiramate had a negligible effect on plasma levels of quetiapine in 7 patients [13]. Unlike carbamazepine, oxcarbazepine does not induce significantly hepatic drug-metabolizing enzymes [24]. In this respect, concomitant administration of oxcarbazepine to patients with bipolar or schizoaffective disorders stabilized on risperidone or olanzapine caused no significant changes in steady-state plasma levels of these two second-generation antipsychotics [26].

\section{CONCLUSION}

Data from a routine TDM service confirm that carbamazepine may cause a significant decrease in plasma concentrations of quetiapine, presumably by inducing CYP3A4. Conversely, steady-state plasma levels of quetiapine were not significantly affected by concomitant administration of therapeutic doses of valproic acid, lamotrigine, topiramate and oxcarbazepine.

\section{ACKNOWLEDGEMENTS}

Supported by grants from the University of Messina (PRA 2005).

\section{REFERENCES}

[2] Sachs, G.; Chengappa, K.N.R.; Suppes, T.; Mullen, J.A.; Brecher, M.; Devine, N.A.; Sweitzer, D.E. Bipolar Disord., 2004, 6, 213.

[3] Calabrese, J.R.; Kech, P.E.; Macfadden, W.; Minkwitz, M.; Ketter T.A.; Weisler, R.H.; Cutler, A.J.; McCoy, R.; Wilson, E.; Mullen, J. Am. J. Psychiatry, 2005, 162, 1351.

[4] Dando, T.M.; Keating, G.M. Drugs, 2005, 65, 2533.

[5] Ichikawa, J.; Li, Z.; Dai, J.; Meltzer, H.Y. Brain Res., 2002, 956, 349.

[6] Devane, C.L.; Nemeroff, C.B. Clin. Pharmacokinet., 2001, 40, 509

[7] Wong, Y.W.J.; Yeh, C.; Thyrum, P.T. J. Clin. Psychopharmacol., 2001, 21, 89.

[8] Grimm, S.W.; Richtand, N.M.; Winter, H.R.; Stams, K.R.; Reele, S.B. Br. J. Clin. Pharmacol., 2005, 61, 58.

[9] Weisler, R.H.; Cutler, A.J.; Ballenger, J.C.; Post, R.M.; Ketter, T.A. CNS Spectr., 2006, 11, 788.

[10] Winter, H.R.; DeVane, C.L.; Figueroa, C.; Ennis, D.J.; HamerMaansson, J.E.; Davis, P.C.; Smith, M.A. Hum. Psychopharmacol. Clin. Exp., 2007, 22, 469.

[11] Castberg, I.; Skogvoll, E.; Spigset, O. J. Clin. Psychiatry, 2007, 68, 1540.

[12] Aichhorn, W.; Marksteiner, J.; Walch, T.; Zernig, G.; Saria, A.; Kemmler, G. Int. Clin. Psychopharmacol., 2006, 21, 81.

[13] Migliardi, G.; D’Arrigo, C.; Santoro, V.; Bruno, A.; Cortese, L.; Campolo, D.; Cacciola, M.; Spina, E. Clin. Neuropharmacol., 2007, 30, 107.

[14] Baumann, P.; Hiemke, C.; Ulrich, S.; Eckermann, G.; Gaertner, I.; Gerlach, M.; Kuss, H.J.; Laux, G.; Muller-Oerlinghausen, B.; Rao, M.L.; Riederer, P.; Zernig, G. Pharmacopsychiatry, 2004, 37, 1.

[15] Avenoso, A.; Facciolà, G.; Salemi, M.; Spina, E. J. Chromatogr. B Biomed. Appl., 2000, 746, 173.

[16] Spina, E.; Perucca, E. Epilepsia, 2002, 43 (Suppl. 2), 37.

[17] Besag, F.M.C.; Berry, D. Drug Saf., 2006, 29, 95.

[18] Hasselstrom, J.; Linnet, K. Ther. Drug Monit., 2004, 26, 486.

[19] Spina, E.; Pisani, F.; Perucca, E. Clin. Pharmacokinet., 1996, 31 198.

[20] Spina, E.; Scordo, M.G.; D’Arrigo, C. Fundam. Clin. Pharmacol., 2003, 17, 517.

[21] Spina, E.; de Leon, J. Basic Clin. Pharmacol. Toxicol., 2007, 100, 4.

[22] Lin, J.H. Pharm. Res., 2006, 23, 1089.

[23] Wen, X.; Wang, J.S.; Rivisto, K.T.; Neuvonen, P.J.; Backman, J.T. Br. J. Clin. Pharmacol., 2001, 52, 547.

[24] Strolin Benedetti, M. Fundam. Clin. Pharmacol., 2000, 14, 301.

[25] Rosenfeld, W.E.; Doose, D.R.; Walker, S.A.; Nayak, R.K. Epilepsia, 1997, 38, 317.

[26] Muscatello, M.R.; Pacetti, M.; Cacciola, M.; La Torre, D.; Zoccali, R.; D’Arrigo, C.; Migliardi, G.; Spina, E. Epilepsia, 2005, 46, 771. 